ÉGYPTE monde arabe

\section{Égypte/Monde arabe}

2| 2005

Les architectures constitutionnelles des régimes

politiques arabes

\title{
Pouvoir exécutif et processus législatif en Égypte
}

\section{Eid Ahmed el-Ghafloul}

\section{(2) OpenEdition}

Journals

Édition électronique

URL : https://journals.openedition.org/ema/1065

DOI : 10.4000/ema.1065

ISSN : 2090-7273

Éditeur

CEDEJ - Centre d'études et de documentation économiques juridiques et sociales

Édition imprimée

Date de publication : 31 décembre 2005

Pagination : 105-132

ISSN : 1110-5097

\section{Référence électronique}

Eid Ahmed el-Ghafloul, «Pouvoir exécutif et processus législatif en Égypte», Égypte/Monde arabe [En ligne], 2 | 2005, mis en ligne le 08 juillet 2008, consulté le 07 juillet 2022. URL : http:// journals.openedition.org/ema/1065; DOI : https://doi.org/10.4000/ema.1065

Ce document a été généré automatiquement le 7 juillet 2022.

Tous droits réservés 


\title{
Pouvoir exécutif et processus législatif en Égypte
}

\author{
Eid Ahmed el-Ghafloul
}

1 Dès l'acquisition de la personnalité internationale par l'Égypte et l'établissement du régime monarchique en 1923, on observe une hégémonie du pouvoir exécutif, notamment des chefs d'État égyptiens, en matière de fixation et de conduite de la politique générale de l'État. Cette hégémonie est encore plus manifeste dans la période qui suit la Révolution de 1952 et l'établissement du régime républicain, qui mit fin aux mécanismes du régime parlementaire qui garantissaient l'existence d'un Parlement dynamique et permettaient aux ministres de participer, avec le roi, à la direction des affaires de l'État.

2 À propos de l'organisation du pouvoir exécutif, la plupart des constitutions égyptiennes (de 1923 à nos jours) ont mis en place une institution gouvernementale homogène, distincte du chef de l'État et seule responsable devant le Parlement de la politique générale de l'État. Ainsi, dans la Constitution actuelle de 1971, le pouvoir exécutif se compose-t-il de deux branches : d'une part, le président de la République qui, malgré son irresponsabilité politique, est doté de pouvoirs très étendus et, d'autre part, le gouvernement, doté de pouvoirs propres et théoriquement responsable devant le Parlement. La Constitution de 1971 reconnaît au gouvernement des pouvoirs propres dans l'élaboration et l'exécution de la politique générale de l'État. Ces textes lui garantissent, en principe, une participation effective dans ce domaine.

Cependant, cette constitution contient d'autres dispositions qui, avec la pratique égyptienne, ont contribué à déformer ce principe bicéphale. D’abord, les dispositions constitutionnelles qui régissent les rapports entre le président de la République et le Conseil des ministres placent ce dernier sous l'autorité du premier. En vertu de l'article 141 de la Constitution de 1971, «le président de la République nomme le président du Conseil des ministres, les vice-présidents du Conseil des ministres, les ministres..., et les décharge de leurs fonctions ». Contrairement à la logique du régime parlementaire, le président n'est pas tenu de choisir ses ministres parmi les chefs d'une majorité parlementaire. Il doit seulement se conformer aux conditions d'âge et de nationalité 
prévues par la Constitution. De là résulte la dépendance des ministres envers lui. Conformément à l'article 142 de la même Constitution, le président de la République a le droit de convoquer le Conseil des ministres, d'assister à ses réunions, et de les présider. Il a également le droit de demander aux ministres de lui présenter des rapports. En outre, au cas où l'Assemblée mettrait en cause la responsabilité du gouvernement, ce dernier ne démissionnerait pas, comme l'exige le régime parlementaire. Le président de la République pourrait mettre en échec la décision de l'Assemblée, s'il veut garder en place un gouvernement qui n'a plus la confiance des parlementaires. À ces dispositions mettant le gouvernement en position d'infériorité par rapport au président de la République, s'ajoute la pratique, qui a beaucoup éloigné le gouvernement du centre de décision, malgré ses compétences déterminées par la Constitution.

\section{Un Parlement faussement bicaméral}

4 Le premier Parlement qu'a connu l'Égypte moderne, en 1866, sous le khédive Ismaïl était monocaméral, composé de la seule «Chambre des notables", dont les compétences étaient purement consultatives. Il devint bicaméral avec la mise en vigueur de la loi organique du $1^{\text {er }}$ mai 1883 , qui prévoyait l'instauration de deux Chambres : le Conseil législatif et l'Assemblée générale. Le Parlement resta bicaméral en 1923, avec une Chambre des députés et un Sénat. Ces deux Chambres étaient alors dotées de compétences équivalentes. Après 1952, les Officiers libres virent dans le monocaméralisme le moyen de maintenir une plus forte unité et d'imposer plus aisément un contrôle partisan. Le Parlement fut donc monocaméral depuis la Constitution de 1956 et jusqu'au 22 mai 1980, où fut instaurée, à la suite d'une révision de la Constitution de 1971, une seconde chambre, l'Assemblée consultative (majlis alshûra) dont les compétences sont purement consultatives.

5 L'Assemblée consultative présente des caractéristiques particulières par rapport à "l'Assemblée du peuple» (majlis al-shacb). Ces caractéristiques tiennent à sa composition et à ses attributions. Elle se compose, conformément à l'article 194 de la Constitution, d'un nombre de membres, déterminé par la loi, mais qui ne doit pas être inférieur à 132. Cette assemblée comprend actuellement 264 membres, dont les deux tiers sont élus au suffrage universel direct tandis que le tiers restant est nommé par le président de la République. La moitié des membres élus doit être composée de paysans et d'ouvriers. Cette condition est également valable pour les membres élus de l'Assemblée du peuple. L'Assemblée consultative suscite de vives critiques en Égypte. En vertu de l'article 198 de la Constitution de 1971, le mandat de ses membres est de six ans. L'élection et la nomination de la moitié des membres interviennent tous les trois ans. L'article 200 de la Constitution interdit le cumul du mandat de cette assemblée et de celui de l'Assemblée du peuple. Conformément à l'article 199 de la Constitution, le président de l'Assemblée consultative et ses deux vice-présidents sont élus par les membres au cours de la première réunion de la session annuelle ordinaire. L'Assemblée consultative ne participe pas au processus législatif, à moins que les avis qu'elle donne au président de la République et à l'Assemblée du peuple puissent orienter l'œuvre législative de ces deux autorités. Elle participe faiblement à la fonction de contrôle parlementaire, sur le fondement des articles 129 et 130 de la Constitution de 1971, qui donnent à ses membres, au même titre qu'à ceux de l'Assemblée législative, le droit de 
demander l'ouverture d'un débat général sur les affaires publiques ou de formuler des vœux sur des questions d'ordre général au président du Conseil des ministres, aux vicepremiers ministres ou aux ministres.

6 Néanmoins, l'Assemblée consultative est dotée de compétences importantes dans le domaine de la presse, considérée par la Constitution de 1971 comme un "pouvoir » de l'État, à la suite de la révision constitutionnelle du 22 mai 1980 et la mise en application de la loi n 148 de 1980 sur le "pouvoir de la presse ». Au-delà de ces compétences, elle exerce deux sortes d'attributions dont la portée est limitée. Son rôle consiste à donner des avis non obligatoires aux pouvoirs publics. Ces attributions sont visées par les articles 195 et 196 de la Constitution. En vertu de l'article 194,

L'Assemblée consultative est chargée d'étudier et de suggérer ce qu'elle juge nécessaire pour sauvegarder les principes des révolutions du 23 juillet 1952 et du 15 mai 1971, pour consolider l'unité nationale et la paix sociale, pour préserver l'alliance des forces laborieuses du peuple et les acquisitions socialistes ainsi que les éléments de base de la société, et ses idéaux, les droits, les libertés et les devoirs publics et pour approfondir le système socialiste démocratique et élargir ses domaines.

Pour remplir cette mission, l'Assemblée peut prendre l'initiative d'aborder l'un des sujets susmentionnés. Elle prépare des rapports qui doivent être communiqués au président de la République ainsi qu'à l'Assemblée du peuple. Quant à l'article 195, il stipule que :

L'Assemblée consultative est requise à donner son avis sur les questions suivantes :

1_ Les propositions portant sur l'amendement d'un ou de plusieurs articles de la Constitution.

${ }^{2}$ - Les projets de lois complétant la Constitution.

3- Le projet du plan général de développement social et économique.

${ }^{4}$ - Les traités de paix et d'alliance ainsi que tous les traités pouvant entraîner une modification des territoires de l'État ou des droits de souveraineté.

5- Les projets de lois qui lui sont soumis par le président de la République.

6- Les questions relatives à la politique générale de l'État ou à sa politique à l'égard des affaires arabes ou étrangères, que le président de la République soumet à l'Assemblée consultative.

L'Assemblée exprimera son opinion à ce sujet par une communication au président de la République et à l'Assemblée du peuple.

7 Cet article distingue entre deux catégories de questions. D'une part, celles prévues par les alinéas 1 à 4 et pour lesquelles la saisine de l'Assemblée consultative est obligatoire ; d'autre part, les questions prévues par les alinéas 5 et 6 , pour lesquelles la saisine de cette Assemblée est facultative pour le président de la République. Le caractère facultatif ou obligatoire de la saisine dépend donc de la nature de la question pour laquelle l'avis de l'Assemblée est demandé. Pour les questions visées par les quatre premiers alinéas de l'article 195 précité, un acte juridique est à accomplir. L'avis de l'Assemblée est donc considéré comme l'une des mesures préparatoires à l'accomplissement de cet acte. L'avis est nécessaire, mais il a toujours une valeur consultative. En revanche, pour les questions prévues par l'alinéa 6, l'avis de l'Assemblée ne porte pas sur une question devant aboutir à un acte juridique. La fonction de l'avis est seulement d'éclairer le président de la République. Le caractère facultatif de la saisine s'impose donc. C'est aussi le cas pour les questions prévues par l'alinéa 5 , bien qu'il puisse s'agir de l'accomplissement d'un acte juridique, d'une loi. Nous pouvons conclure, avec l'ensemble des publicistes égyptiens que, pour les questions dont l'Assemblée consultative est obligatoirement saisie, l'avis de celle-ci, 
malgré son caractère non contraignant, constitue une condition de forme nécessaire pour la validité de l'acte juridique à édicter.

8 Nous pouvons donc facilement constater que, sous la Constitution de 1971 actuellement en vigueur, le Parlement égyptien est monocaméral, composé de la seule « Assemblée du peuple", titulaire de la compétence législative générale et seule habilitée à contrôler politiquement le gouvernement. Cette assemblée est, cependant, totalement soumise au président de la République, de façon à réduire considérablement son pouvoir de contrôle et à orienter son œuvre législative au service de sa politique tant intérieure qu'extérieure. Elle est, en effet, en situation de dépendance à l'égard du président de la République, que ses interventions dans l'exercice de la fonction législative de l'État font apparaître comme un législateur parallèle.

\section{La dépendance du Parlement à l'égard du chef de l'État}

9 Les Constitutions successives de l'Égypte républicaine ont mis à la disposition du Parlement de nombreux moyens pour contrôler l'action du pouvoir exécutif. Les parlementaires ont tout d'abord le droit de s'informer et de surveiller l'action de l'exécutif. Ils peuvent ainsi formuler des questions, écrites ou orales, auxquelles les membres du gouvernement doivent répondre. Ensuite, le Parlement peut, à tout moment, constituer des commissions d'enquête et de contrôle, afin d'accueillir des éléments d'information sur des questions déterminées se rattachant à l'intérêt général . Enfin, depuis la Constitution de 1964, le Parlement peut mettre en jeu la responsabilité politique du gouvernement. Cependant, tous ces moyens sont inefficaces, compte tenu de la concentration des pouvoirs entre les mains du président de la République, chef incontesté du pouvoir exécutif, principal acteur de la politique générale de l'État et disposant de nombreux moyens pour dominer le Parlement et orienter ses activités au service de sa propre politique.

10 Les Constitutions de l'Égypte républicaine consacrent nettement, en effet, la suprématie du président de la République sur le Parlement. Les moyens les plus importants d'action directe sur le Parlement, qui limitent considérablement le pouvoir de contrôle de ce dernier, tiennent surtout à la présidence par le chef de l'État du parti dominant au sein du Parlement. Dans le cadre du processus entamé par les révolutionnaires de 1952 pour la liquidation de l'ancien régime, la dissolution des partis politiques fut proclamée par le décret du 16 janvier 19532. Pour combler le vide qui en résultait et pour assurer aux Officiers libres l'appui nécessaire des masses populaires, la création du « Rassemblement de la libération » fut annoncée le 23 janvier 1953.

11 L'absence d'une idéologie politique définie et d'une structure précise entraînèrent l'échec puis la disparition de ce rassemblement. Il fut, en effet, remplacé, le 29 mai 1957, par 1'» Union nationale » prévue par l'article 192 de la Constitution de 1956 et formée par les citoyens « [...] en vue de réaliser les objectifs de la Révolution et afin de conjuguer leurs efforts pour édifier une nation saine des points de vue politique, social et économique ». Le fonctionnement de l'Union nationale s'avéra également un échec. Il incita Nasser, alors président de la République, à présenter au « Congrès national des forces populaires », le 21 mai 1962, le projet de «Charte nationale ». Celle-ci prévoyait la création d'une nouvelle organisation partisane, l'» Union socialiste arabe», 
consacrée ultérieurement par l'article 5 de la Constitution de 1971. Cette formation politique unique monopolisa la vie politique égyptienne jusqu'en 1976, lorsque le pays connut une nouvelle phase : celle du multipartisme octroyé et strictement contrôlés. En 1976, Sadate voulut apparaître comme le rénovateur de la vie politique égyptienne. Ainsi, le 11 novembre de la même année, dans son discours devant l'Assemblée du peuple, évoqua-t-il la possibilité de la formation de partis politiques. À cet effet fut promulguée la loi ${ }^{\circ} 40$ du 2 juillet 1977 sur les partis, modifiée ultérieurement en 1979 et en 1980. L'article 5 de la Constitution de 1971 a été également révisé, disposant que :

Le système politique de la République arabe d'Égypte est basé sur la multiplicité des partis dans le cadre des éléments de base et des principes fondamentaux de la société égyptienne stipulés dans la Constitution.

Les dispositions légales concernant l'organisation des partis politiques, ainsi que les dispositions des lois électorales, réduisent la chance d'avoir une opposition suffisante au sein du Parlement et écartent donc la possibilité d'une alternance politique. Elles garantissent au seul Parti national démocratique, gouvernemental, une position très confortable au sein de l'Assemblée. En effet, le Parti national démocratique disposait de $85 \%$ des sièges dans le Parlement élu en 2000. Ce parti est, comme c'était le cas du parti unique, présidé par le chef de l'État. Ainsi le Rassemblement de la libération, créé en 1953, était-il présidé par le général Mohamed Naguib, alors président de la République. Nasser, lui, assumait les fonctions de secrétaire générals. De même, pour l'Union socialiste arabe, créée en 1962, Nasser puis Sadate, à partir de 1971, se désignèrent euxmêmes comme présidents. Le Parti national démocratique fut présidé de 1976 à 1981 par Sadate. Le président de la République, disposant toujours d'une très forte majorité qu'il contrôle, peut ainsi, s'il le veut, modifier la Constitution et il pouvait, jusqu'à la réforme constitutionnelle de mai 2005 , se faire réélire pour de nouveaux sextennats, puisque l'Assemblée du peuple avait l'initiative du choix du chef de l'État, ce choix étant toutefois soumis à référendum. Dans les deux cas, une majorité de deux tiers était exigée. Cette large majorité soutient, avec une remarquable constance, le pouvoir exécutif et notamment le chef de l'État, dont les orientations en matière de politique générale deviennent hors de portée des critiques de la majorité par les parlementaires.

13 Le chef de l'État peut aussi dissoudre le Parlement, ce droit est consacré par les constitutions égyptiennes successives ${ }^{6}$. Ce droit est actuellement organisé par l'article 136 de la Constitution de 1971. D'après celui-ci :

Le président de la République ne peut dissoudre l'Assemblée du peuple qu'en cas de besoin et après un référendum populaire. Dans ce cas, le président de la République rend un décret portant suspension des séances de l'Assemblée et fixant le référendum dans un délai de trente jours. Au cas où la majorité absolue des votes émis approuve la dissolution, le président de la République promulgue un décret à cet effet.

Le décret portant convocation des citoyens à de nouvelles élections de l'Assemblée $\mathrm{du}$ peuple doit fixer un délai ne dépassant pas les soixante jours à partir de la date de la proclamation du résultat du référendum.

La nouvelle Assemblée se réunit dans les dix jours suivant le scrutin ${ }^{7}$.

14 L'Assemblée du peuple peut être également considérée comme dissoute dans le cas d'un consentement populaire à la suite d'un référendum sollicité par le président de la République, au cas où elle décide de mettre en cause la responsabilité politique du gouvernement ${ }^{8}$. En droit égyptien, le droit de dissolution a pour fonction de renforcer les pouvoirs du président de la République face au Parlement et de renouveler la confiance du peuple au chef de l'État. Ainsi, le 19 avril 1979, le chef de l'État a-t-il 
dissous l'Assemblée du peuple après acceptation par le corps électoral à la fois du traité de paix avec Israël et des mesures de « renforcement de la démocratie en Égypte ». Le but de cette dissolution était, pour lui, de trouver une Assemblée plus souple, plus compréhensive de sa nouvelle politique et de faire approuver l'immunité de son régime. Par cette dissolution, il s'est débarrassé des parlementaires hostiles à sa politique, notamment extérieure ${ }^{9}$.

Par ailleurs, le président de la République peut, «en cas de besoin », conformément à l'article 204 de la Constitution, dissoudre l'Assemblée consultative instituée à la suite de la révision constitutionnelle du 22 mai 1980. Ainsi placé à la merci de celui-ci, le Parlement ne peut pas exercer un contrôle efficace sur l'action du pouvoir exécutif et, plus particulièrement, sur la politique étrangère du président dont «le droit de dissolution discrétionnaire est un formidable moyen de pression sur l'Assemblée, au cas où celle-ci voudrait contester sa politique ${ }^{10}$.

Toutefois, sans en arriver à une dissolution, le pouvoir exécutif dispose de divers moyens pour influencer le fonctionnement du Parlement. En effet, c'est au gouvernement qu'il revient de fixer l'ordre du jour de l'Assemblée du peuple ${ }^{11}$, à laquelle les ministres ont le droit d'accès et de parole, d'autant plus qu'ils peuvent cumuler entre leur fonction de ministre et un mandat parlementaire ${ }^{12}$. À cela, on peut ajouter les grandes facilités dont bénéficient les projets de lois d'origine gouvernementale pour être discutés et votés par l'Assemblée. De plus, le président de la République dispose, à lui seul, de plusieurs moyens lui permettant d'influencer le Parlement et d'orienter son activité au service de sa propre politique, notamment étrangère. En effet, il nomme, depuis 1964, dix membres dans l'Assemblée du peuple, il a le droit de prononcer des discours devant celle-ci et il peut opposer son veto aux lois votées par l'Assemblée.

Sous le régime de la Constitution de l'Égypte monarchique, le monarque avait le droit de nommer les deux cinquièmes des sénateurs, mais les députés devaient être tous élus au suffrage universel. La même solution fut retenue par les constitutions républicaines de 1956 et de 1964. Sous celles-ci, la totalité des membres devait être élue au suffrage universel et secret ${ }^{13}$. Sous la Constitution de 1971, le Parlement égyptien est composé d'une Chambre unique, l'Assemblée du peuple, à côté de laquelle existe, depuis 1980, l'Assemblée consultative. Sur les 454 députés que comporte actuellement l'Assemblée du peuple, dix sont nommés par le président de la République. Selon le pouvoir exécutif, cette solution permet au président de la République d'envoyer à l'Assemblée des membres de très haut niveau et d'une expérience suffisante qui leur permettent, tout en restant au-dessus de la concurrence politique, de défendre les intérêts des tendances politiques dont les résultats aux élections sont trop faibles pour qu'elles soient représentées au Parlement. Néanmoins, la nomination de ces dix députés suscite des critiques en Égypte, qui y voient, contrairement aux prétentions de l'exécutif, une violation du système représentatif et un moyen de plus pour dominer le Parlement. Les membres nommés ne pourraient contrer la politique du président de la République, même si elle n'était pas en accord avec l'intérêt général du pays ${ }^{14}$.

Le droit de message est un droit du chef de l'État à communiquer au Parlement ou éventuellement à la nation afin de faciliter la collaboration des pouvoirs. Il lui permet, d'ailleurs, de participer personnellement à la conduite des affaires publiques. En droit égyptien, le droit de message est consacré par l'ensemble des constitutions 
égyptiennes, de 1923 jusqu'à nos jours ${ }^{15}$. La Constitution monarchique de 1923 stipulait dans son article 42 que :

Le roi ouvre la session ordinaire du Parlement par un discours du trône adressé aux deux chambres réunies et exposant la situation du pays. Chacune des deux chambres présentera une adresse en réponse à ce discours.

Actuellement, l'article 132 de la Constitution de 1971 dispose que :

Le président de la République inaugure la session ordinaire de l'Assemblée du peuple par un discours exposant la politique générale de l'État. Il a également le droit de traiter d'autres sujets devant l'Assemblée. Il appartient à cette dernière de discuter de l'exposé du président de la République.

Outre le droit de discours organisé par les textes, le chef de l'État peut, chaque fois qu'il le juge nécessaire, faire un discours devant le Parlement. Actuellement, il exerce souvent ce droit dans les occasions d'envergure nationale en rappelant aux parlementaires leurs missions ${ }^{16}$. À l'époque monarchique comme à l'époque républicaine, le droit de message a offert aux chefs de l'État l'occasion de tracer les grandes lignes de leur politique intérieure et extérieure. De l'époque républicaine à nos jours, le droit de message est considéré comme un moyen d'action capital entre les mains du président de la République. Il lui permet d'exercer une pression sur la volonté des représentants du peuple et sur l'opinion publique. Ses discours, diffusés par tous les moyens d'information lus, écoutés ou regardés, déterminent les options du Parlement. La pratique égyptienne atteste d'ailleurs que les plus grandes décisions de politique étrangère sont prises dans des discours de ce genre, énoncés dans ou en dehors du Parlement. Par exemple, la suppression en 1951 du traité d'alliance égypto-britannique de 1936, la nationalisation du canal de Suez par Nasser en 1956 et la décision de Sadate de se rendre à Jérusalem en 1977.

21 À l'occasion de la promulgation des lois, le chef de l'État peut recourir à l'exercice d'un autre pouvoir : en effet, il peut, au lieu d'ordonner la promulgation de la loi, demander une seconde délibération sur tout ou partie du texte concerné. Ce droit était organisé par les constitutions de l'Égypte monarchique sous forme de sanction royale. Il permettait au monarque de contrecarrer la volonté du Parlement en faisant obstacle au vote de tout projet de loi qu'il n'approuvait pas ${ }^{17}$. Il est reconduit dans toutes les constitutions de l'Égypte républicaine depuis 1956, sous forme d'un veto suspensif, à l'instar $d u$ modèle américain résultant du principe d'égalité des pouvoirs ${ }^{18}$. Actuellement, le veto suspensif est organisé par les articles 112 et 113 de la Constitution du 11 septembre 1971. L'article 112 prévoit que : «le président de la République a le droit de promulguer les lois ou d'y opposer son veto ". D'après l'article 113, si le président oppose son veto à un projet de loi adopté par l'Assemblée, il le retourne dans un délai de 30 jours à partir de la date où ce projet de loi lui a été soumis. Au cas où le projet de loi ne serait pas retourné à l'Assemblée dans le délai, il est considéré comme une loi et promulgué. S'il est retourné à l'Assemblée dans le délai susmentionné et voté à la majorité des deux tiers de ses membres, il est considéré comme une loi et promulgué. Le veto présidentiel, attribution classique du chef de l'État parlementaire, devient « une arme politiquement efficace au fur et à mesure que ce parlementarisme fait place à un régime qui, combinant les traits du régime parlementaire et du régime présidentiel, affirme la prépondérance de l'exécutif sur le Parlement ${ }^{19}$ ", comme c'est le cas en Égypte. 


\title{
L'autonomie de l'exécutif vis-à-vis du Parlement
}

Comme nous l'avons déjà vu, les constitutions de l'Égypte républicaine ont donné des pouvoirs considérables au chef de l'État qui les exerce effectivement sans que ses actes ne soient soumis à la règle du contreseing ministériel. Or, malgré l'extension de ses pouvoirs, le chef de l'État ne peut être tenu pour responsable devant le Parlement qu'en cas de haute trahison. Ainsi, le principe de l'irresponsabilité politique du chef de l'État, qui a une origine monarchique en droit égyptien, s'applique également au président de la République qui détient et exerce le pouvoir exécutif. Sous le régime de la Constitution actuelle de 1971, le principe de l'irresponsabilité politique du président de la République est implicitement consacré par l'article 85 qui parle seulement d'une éventuelle mise en accusation pénale. Il dispose que :

\begin{abstract}
La mise en accusation du président de la République pour haute trahison ou pour avoir commis un crime s'effectue sur une motion présentée par le tiers au moins des membres de l'Assemblée du peuple. L'acte d'accusation n'est valable que s'il est adopté par une majorité des deux tiers des membres de l'Assemblée.

Dès que la décision de mise en accusation est prise, le président de la République est suspendu de ses fonctions qui sont assumées provisoirement par le vice-président de la République, jusqu'à ce qu'il soit statué sur l'accusation. Le président de la République sera jugé par une cour spéciale dont la composition, la procédure du jugement et la peine à infliger seront déterminées par une loi. En cas de condamnation, il sera déchargé de ses fonctions, sans préjudice des autres peines ${ }^{20}$.
\end{abstract}

La composition de la Cour spéciale, chargée de juger le président de la République, ainsi que la procédure du jugement sont déjà fixées par la loi n 247 de 1956. Cependant, la soumission totale du Parlement au président de la République rend impensable toute accusation de ce dernier par les parlementaires ${ }^{21}$. L'irresponsabilité absolue du président et l'extension de ses pouvoirs, qu'il exerce effectivement, entraînent des conséquences négatives sur le fonctionnement du système politique égyptien en général et, en particulier, en matière de la politique étrangère, dans la mesure où il alors est difficile d'en contrôler les auteurs.

Bien évidemment, l'autonomie de l'exécutif vis-à-vis du Parlement entraîne également l'autonomie du gouvernement. Les ministres étaient responsables individuellement et à la fois devant l'Assemblée nationale et le président de la République sous les Constitutions de 1956 et de 1958 qui avaient rompu avec le régime parlementaire suite à la Révolution de 1952. Mais, à partir de l'entrée en vigueur de la Constitution de 1964, qui marque un retour vers le régime parlementaire, les ministres deviennent - en sus de la responsabilité individuelle de la gestion de leurs ministères - responsables collectivement, devant l'Assemblée, de la politique générale de 1'État. Le principe de la responsabilité collective des ministres, ainsi consacré par la Constitution de 1964, a été repris par celle de 1971 actuellement en vigueur. Ce principe est cependant déformé, puisque les mécanismes prévus pour la mise en œuvre de cette responsabilité sont tels qu'ils donnent au président de la République la décision finale en la matière.

La Constitution de 1971 consacre le principe de la responsabilité pénale des ministres à côté de leur responsabilité politique, soit individuelle soit collective, conformément à l'article 126, prévoyant que : "Les ministres sont responsables devant l'Assemblée du peuple de la politique générale de l'État. Chaque ministre est responsable des affaires de son département [...]». En vertu de l'article 128/1 de la même Constitution ${ }^{22}$, « au cas où l'Assemblée retire sa confiance à un vice-Premier ministre, à un ministre ou à 
l'un de leurs suppléants, il doit démissionner de ses fonctions $»^{23}$. La procédure de responsabilité individuelle peut être ainsi engagée contre tout membre du gouvernement, à l'exception du président du Conseil des ministres, auquel s'appliquent les dispositions particulières de la responsabilité collective. Celle-ci est organisée de deux manières par les articles 133 et 127. D'une part, la question de confiance, selon laquelle le gouvernement peut prendre l'initiative d'engager sa responsabilité devant l'Assemblée du peuple. D'autre part, la motion de censure, en vertu de laquelle l'Assemblée pourra prendre l'initiative de censurer le gouvernement qui n'a plus sa confiance. La question de confiance n'est pas expressément prévue par les textes mais elle découle indirectement, d'après une partie de la doctrine, de l'esprit de l'article 133 de la Constitution ${ }^{24}$ ainsi rédigé :

Le président du Conseil des ministres présente le programme de son gouvernement, après la formation de celui-ci et lors de l'inauguration de la session ordinaire de

l'Assemblée du peuple. L'Assemblée du peuple engage le débat sur ce programme.

Il semble logique, pour cette partie de la doctrine, que le président du Conseil des ministres puisse, s'il le veut, engager la responsabilité de son gouvernement sur son programme lors de l'inauguration de la session ordinaire de l'Assemblée du peuple ${ }^{25}$. La motion de censure est explicitement prévue par l'article 127, alinéas I et 2 de la Constitution de 1971 :

L'Assemblée du peuple peut, à la demande du dixième de ses membres, mettre en cause la responsabilité du président du Conseil des ministres. La décision à ce sujet doit être prise à la majorité des membres de l'Assemblée. Cette décision ne peut être prise qu'après une interpellation adressée au gouvernement et trois jours au moins à partir de la présentation de la demande.

Ainsi, après une interpellation, l'Assemblée du peuple peut-elle mettre en cause la responsabilité du président du Conseil des ministres par un vote à la majorité de ses membres. Ce faisant, elle met collectivement en cause le gouvernement, car les ministres sont responsables de la politique générale de 1'État - une politique que représente le chef du gouvernement dont ils sont alors solidaires ${ }^{26}$.

D'après les mécanismes du régime parlementaire, en cas de censure ou de vote de confiance, le gouvernement tout entier est obligé de remettre directement sa démission. Or, il n'en est rien en droit égyptien, car la Constitution de 1971 multiplie les obstacles à cette démission, en attribuant au président de la République la possibilité de mettre en échec la décision de l'Assemblée. La Constitution de 1971 prévoit, en effet, un rôle actif, voire décisif, du président de la République dans la procédure de mise en jeu de la responsabilité politique du gouvernement. Ce rôle intervient après la décision finale de l'Assemblée mettant en cause cette responsabilité après une censure ou un vote de confiance. L'intervention du président de la République est clairement consacrée par les alinéas trois à six de l'article 127 précité. Ils sont ainsi rédigés :

[...] Au cas où la responsabilité serait établie, l'Assemblée prépare un rapport qu'elle soumet au président de la République comportant les éléments de la question, ainsi que son avis et ses motifs. Le président de la République peut retourner ce rapport à l'Assemblée dans un délai de dix jours.

Si l'Assemblée adopte de nouveau le rapport, le président de la République peut soumettre le conflit entre l'Assemblée et le gouvernement à un référendum dans les trente jours à partir de la date du dernier vote de l'Assemblée. Dans ce cas, les séances de l'Assemblée sont suspendues.

Si le résultat du référendum est favorable au gouvernement, l'Assemblée sera considérée comme dissoute. Dans le cas contraire, le président de la République accepte la démission du gouvernement ${ }^{27}$. 
La procédure de l'article 127 offre au chef de l'État un moyen efficace de défendre sa propre politique extérieure. Il est, comme nous l'avons vu, le principal acteur de la politique étrangère et sa responsabilité ne peut être engagée devant l'Assemblée. Celle$\mathrm{ci}$, si elle veut mettre en cause la politique étrangère, doit user des moyens mis à sa disposition c'est-à-dire, entre autres, l'engagement de la responsabilité du gouvernement. Mais, le chef de l'État, voyant sa politique mise indirectement en cause, peut faire échec à la décision de l'Assemblée. Pour ce faire, il dispose de deux moyens : soit demander à l'Assemblée de revoir sa décision soit, si cette dernière maintient sa position, soumettre la question au peuple. Dans cette dernière hypothèse, c'est l'Assemblée qui sera considérée comme dissoute, puisque le «oui» en faveur du président de la République dans les référendums à l'égyptienne a toujours été de plus de $95 \%$.

\section{Les interventions du chef de l'État dans le domaine législatif}

31 Toutes les constitutions égyptiennes ont prévu différentes formes d'interventions $d u$ pouvoir exécutif dans le domaine de la compétence législative du Parlement. Ces interventions, en temps normal aussi bien qu'en temps de crise, n'influencent pas seulement la fonction législative de ce dernier mais également son pouvoir de contrôle. En temps normal, elles prennent trois formes. D'abord, les règlements d'exécution des lois, pris par le chef de l'État, sans les modifier ou en suspendre l'exécution. Ensuite, les règlements autonomes, dont l'auteur est également le chef de 1'État et dont l'objet est la création et l'organisation des services publics. La troisième forme concerne les règlements de police qui, au nom du maintien de la tranquillité et de la salubrité publique, peuvent apporter des restrictions importantes aux libertés des individus. Les règlements de police sont consacrés explicitement par les constitutions républicaines. Quant à la Constitution monarchique de 1923, elle ne les prévoyait pas, mais le roi les pratiquait. À ces trois formes, on peut ajouter une quatrième, propre aux constitutions républicaines: le référendum permet au président de la République de dépasser le Parlement par les consultations populaires, en temps normal comme en temps de crise. De plus, en période d'exception, les constitutions égyptiennes ont prévu d'autres formes d'interventions du chef de l'État dans le domaine de la compétence parlementaire. En effet, dans des circonstances exceptionnelles, celui-ci peut rendre des décrets ayant force de loi, soit sur habilitation expresse du Parlement, soit en cas d'absence de ce dernier.

En temps de crise, le président de la République peut, en effet, remplacer le Parlement et régler, par la voie de décrets-lois, des questions relevant normalement de la compétence de ce dernier. Ces interventions reposent soit sur une habilitation expresse du Parlement, soit sur une habilitation accordée directement par la Constitution. Dans 
la pratique égyptienne, une interprétation excessive des dispositions constitutionnelles a permis l'extension des interventions du président de la République par des décrets de délégation, des décrets de nécessité ou par les décrets de crise spéciaux de l'article 74 de la Constitution, dans le domaine de la compétence législative de l'Assemblée du peuple.

La Constitution de l'Égypte monarchique n'autorisait pas le Parlement à déléguer ses compétences au pouvoir exécutif. La thèse dominante en doctrine et dans la jurisprudence était qu'en l'absence de textes une telle délégation ne pouvait avoir lieu. Pourtant, il y eut des cas, très rares, où le Parlement autorisa le pouvoir exécutif à légiférer par le moyen des décrets ayant force de loi dans des matières importantes. Cela a été le cas de la loi $n^{\circ} 2$ de 1930 relative aux tarifs douaniers et de la loi $n^{\circ} 3$ de la même année, relative à la taxe de production ${ }^{29}$. Quant aux constitutions de l'Égypte républicaine, elles ont consacré expressément cette délégation législative. Depuis la Constitution de 1956, le Parlement égyptien peut, sous certaines conditions, déléguer ses compétences législatives au président de la République. La délégation législative est actuellement régie par l'article 108 de la Constitution de 1971, ainsi rédigé :

Le président de la République peut en cas de nécessité et dans des circonstances exceptionnelles, en vertu d'une délégation des pouvoirs qui lui est conférée par l'Assemblée du peuple à la majorité des deux tiers de ses membres, rendre des décrets ayant force de loi. La délégation des pouvoirs doit être accordée pour une durée déterminée et indiquer les objets des décrets et les bases sur lesquelles ils reposent. Ces décrets doivent être soumis à l'Assemblée du peuple au cours de la première réunion qu'elle tiendra après l'expiration du délai de cette délégation. $\mathrm{Au}$ cas où ces décrets ne sont pas soumis à l'Assemblée ou lui ont été soumis et ont été rejetés, ils cessent d'avoir force de loi $^{30}$.

Cet article appelle les observations suivantes :

- Le seul bénéficiaire de cette délégation de compétence législative est le président de la République, qui ne peut pas à son tour déléguer cette compétence à une autorité inférieure ${ }^{31}$.

- L'initiative de la loi de délégation, considérée loi comme les autres, appartient non seulement au président de la République, mais également aux parlementaires qui peuvent, dans les conditions posées par cet article, proposer la délégation de la compétence du Parlement au président de la République ${ }^{32}$.

- La délégation législative peut avoir pour objet toutes les matières relevant de la compétence du Parlement en vertu des dispositions constitutionnelles ${ }^{33}$.

- Les décrets présidentiels, pris sur la base de l'article 108, une foispromulgués dans les conditions exigées, doivent être soumis à l'Assemblée du peuple au cours de la première réunion qu'elle tiendra après l'expiration du délai de cette délégation. Au cas où ces décrets ne seraient pas soumis à l'Assemblée, ou lui auraient été soumis puis rejetés, ils cessent d'avoir force de loi. Ces décrets disparaîtront donc de « l'ordonnancement juridique », parce qu'ils ne sont pas considérés comme des lois mais comme des actes administratifs. Et, en cette qualité, ils ne peuvent plus régler une matière réservée à la loi.

- En l'absence d'une solution explicite dans l'article 108, la cessation dela force juridique des décrets de délégation ne concernera que l'avenir. Elle n'aura aucun effet rétroactif ${ }^{34}$.

À côté du contrôle parlementaire, la délégation législative déclenche deux sortes de contrôle juridictionnel. D'abord, le contrôle de la légalité administrative auquel sont soumis les décrets présidentiels avant leur approbation par le Parlement puis le contrôle a posteriori de constitutionnalité de la loi de délégation elle-même et des 
décrets-lois pris sur son fondement une fois approuvés par le Parlement ${ }^{35}$. Les décrets de délégation de l'article 108 peuvent donc, à la suite d'une désapprobation parlementaire ou d'une décision juridictionnelle, perdre leur effet pour l'avenir. Pour que le président de la République, ou probablement les parlementaires, puissent demander la mise en œuvre de l'article 108, trois conditions doivent être réunies. D'abord, des circonstances exceptionnelles, exigeant la rapidité d'action par le moyen de décrets-lois en application de la théorie de la nécessité ${ }^{36}$, puisque le principe est, qu'en temps normal, le Parlement ne peut déléguer les compétences qu'il reçoit de la Constitution $^{37}$. En second lieu, la loi de délégation doit limiter la délégation législative dans le temps. Ainsi ne suffit-il pas de déterminer cette durée par des expressions générales et vagues - comme il résulte des lois habilitant, depuis 1972, le président de la République à ratifier les accords relatifs aux armements ${ }^{38}$. La loi de délégation doit, enfin, indiquer l'objet des décrets-lois et les bases sur lesquelles ils reposent. L'article 108 ne permet, en effet, qu'une délégation limitée. Par conséquent, une délégation générale accordée par une seule loi est inconstitutionnelle.

La pratique égyptienne révèle un recours fréquent à la procédure de l'article 108 dans les domaines les plus importants. Le recours à cet article a toujours été justifié par les circonstances exceptionnelles résultant de l'état de guerre développé depuis l'agression israélienne du 5 juin 1967. Cependant, la signature du traité de paix égypto-israélien en 1979 n'a pas permis, jusqu'à nos jours, de mettre fin à cette pratique. Il apparaît donc que le système politique et constitutionnel égyptien porte en lui-même les germes d'une crise permanente.

Toutes les constitutions égyptiennes, depuis celle de 1923 jusqu'à celle de 1971, ont permis, en outre, au chef de I'État de prendre des décrets de nécessité, pour faire face à «des événements survenant qui exigent de recourir à des mesures urgentes qui ne souffrent pas de retard $»^{39}$. Durant la période monarchique, les décrets de nécessité étaient gérés par l'article 41 de la Constitution de 1923. Ils sont actuellement prévus et organisés par l'article 147 de la Constitution de 1971 :

Si, en l'absence de l'Assemblée du peuple, des événements surviennent qui exigent de recourir à des mesures urgentes ne souffrant pas de retard, le président de la République peut prendre à leur sujet des décrets ayant force de loi. Ces décrets doivent être soumis à l'Assemblée du peuple dans les quinze jours, à partir de la date à laquelle ils ont été pris, si l'Assemblée est en session, et à la première réunion de l'Assemblée en cas de dissolution ou de suspension de ses séances. S'ils ne lui sont pas soumis, ils perdent, rétroactivement, la force de loi qu'ils avaient, sans nécessité de prendre de décision à ce sujet. Mais s'ils sont soumis à l'Assemblée et si celle-ci les rejette, ils perdent, rétroactivement, la force de loi qu'ils avaient, à moins que l'Assemblée n'en approuve l'exécution pour la période précédente ou ne décide d'en régler autrement les effets.

Cet article pose deux séries de conditions pour la mise en œuvre de la procédure qu'il met en place. Ces conditions concernent, d'une part, le recours à la procédure de l'article 147 et, d'autre part, la validité des décrets pris sur son fondement ${ }^{40}$. Pour le recours à la procédure de l'article 147 par le président de la République, deux conditions sont nécessaires. D'abord, l'existence de circonstances exceptionnelles. D'après une doctrine établie, il appartient au président de la République de décider si ces circonstances méritent ou non de recourir à la procédure de l'article 147, sous le contrôle postérieur de l'Assemblée du peuple ${ }^{41}$. À côté du contrôle parlementaire, un contrôle juridictionnel de l'existence de ces circonstances est exercé, d'abord par la juridiction administrative pour la légalité des décrets de nécessité avant leur 
ratification par le Parlement, dans la mesure où ils sont considérés comme des actes administratifs et, en second lieu, par le juge constitutionnel, après leur approbation par le Parlement, dans la mesure où cette ratification leur donne le caractère d'actes législatifs ${ }^{42}$. La seconde condition de recours à l'article 147 concerne l'absence du Parlement. À cet égard, les constitutions égyptiennes ont connu une évolution manifeste. Pendant la période monarchique, dans la Constitution de 1923, le roi ne pouvait recourir aux décrets de nécessité que dans l'intervalle des sessions parlementaires. La Constitution de 1930 rajouta la période de dissolution ${ }^{43}$, suivie en cela par les deux constitutions républicaines de 1956 et de 1964. La Constitution de 1971, dans son article 147, a étendu la possibilité pour le président de la République de recourir aux décrets de nécessité. L'expression utilisée est celle de 1'»absence de l'Assemblée du peuple ». Cet article peut donc être mis en œuvre dans l'intervalle des sessions parlementaires, pendant la dissolution du Parlement et en cas de suspension des séances de ce dernier ${ }^{44}$.

Pour la validité des décrets de nécessité, deux conditions sont exigées par l'article 147. Ces conditions concernent le fond et la forme de ces décrets ${ }^{45}$. Les mesures prises par le chef de l'État doivent être effectivement nécessaires pour faire face aux circonstances exceptionnelles. Ensuite, tout comme pour les décrets de délégation de l'article 108, les décrets de nécessité doivent être soumis à l'approbation de l'Assemblée du peuple. Mais à l'encontre de l'article 108, l'article 147 pose des délais différents. En effet, une distinction est faite entre deux hypothèses. La première est celle où le Parlement est en fonctionnement régulier. Selon cette hypothèse, il faut déposer les décrets dans un délai de quinze jours à compter du jour de leur promulgation. Le second cas est celui où le Parlement est dissous ou suspendu. Là, il faut que les décrets soient déférés au Parlement à sa première réunion, après les nouvelles élections ou après la fin de la suspension. Lorsque les décrets de nécessité sont promulgués dans les conditions exigées par la Constitution, ils peuvent abroger ou modifier les lois antérieures. Mais, à l'encontre des décrets de délégation, s'ils sont désapprouvés par l'Assemblée, ils perdent rétroactivement leur force de loi, faute d'une autorisation parlementaire préalable ${ }^{46}$.

La pratique égyptienne atteste également d'un recours injustifié à l'article 147 pour faire face à des circonstances pouvant être régies par des mesures normales. Cet article est, cependant, peu utilisé par le président de la République, qui préfère recourir à l'article 74. Le contrôle est alors plus limité ${ }^{47}$ et cette disposition donne au président des pouvoirs très étendus pour faire face au «[...] danger menaçant l'unité nationale, ou la sécurité de la patrie ou empêchant les institutions de l'État de remplir leur rôle constitutionnel [...] ». À l'instar de l'article 16 de la Constitution française de 1958, l'article 74, qui est une innovation de la Constitution de 1971, a été jusqu'à maintenant utilisé à trois reprises ${ }^{48}$. Mais, en aucun cas, il n'a été utilisé en matière de conclusion des traités. Ce qui est peut-être dû au fait que les périodes de sa mise en œuvre sont assez courtes. L'article 74 crée une sorte de confusion et de concentration de pouvoirs très étendues, aussi bien sur le plan interne que sur le plan international, entre les mains du président de la République. Les mesures prises par celui-ci dans le cadre de cet article touchent bien évidemment à des matières relevant de la compétence législative de l'Assemblée du peuple et constituent, par conséquent, une immixtion dans la fonction législative de l'Assemblée. Il est, en effet, ainsi tourné :

En cas de danger menaçant l'unité nationale ou la sécurité de la patrie, ou empêchant les institutions de l'État de remplir leur rôle constitutionnel, il 
appartient au président de la République de prendre les mesures urgentes pour parer à ce danger. Dans ce cas, il adresse un message au peuple et il est procédé à un référendum sur les mesures qu'il aura prises dans les soixante jours qui suivent.

\section{Le pouvoir référendaire du chef de l'État}

41 En droit égyptien, le référendum constitue une véritable intervention dans le domaine de compétence de l'Assemblée. L'article 152 de la Constitution de 1971 stipule que : «Le président de la République peut consulter le peuple sur les questions se rattachant aux intérêts supérieurs du pays $»^{49}$. Cet article donne une définition très large du champ d'application de la procédure qu'il met en place. En effet, toute question se rattachant aux intérêts supérieurs du pays peut faire l'objet d'une consultation populaire ${ }^{50}$. Le seul critère retenu par ce texte est l'importance de la question. Le président de la République est le seul en mesure d'estimer celle-ci. Le droit de mettre en œuvre cette procédure lui appartient exclusivement. Il en résulte que l'article 152 pourrait être appliqué lorsque le président de la République se trouve face à des questions sur lesquelles des décisions importantes doivent être prises. Ces questions peuvent être relatives à la vie sociale, économique et culturelle interne du pays, à ses relations internationales ou à son adhésion à des organisations supranationales ${ }^{51}$.

En 1979, l'article 152 fut mis en application pour approuver le traité de paix égyptoisraélien du 26 mars de la même année. Mais, avant d'être utilisé en matière de traités internationaux, cet article a été employé à deux reprises pour l'obtention d'une « couverture » populaire de la politique générale du président Sadate après la guerre d'octobre 1973, qui fit de lui un héros national. Sa première application date du 15 mai 1974, pour l'approbation du «document d'octobre» comportant, entre autres, l'introduction en Égypte des mécanismes de l'économie de marché et adopté à la majorité de 99,95 \% des voix exprimées ${ }^{52}$. Il a été utilisé, en second lieu, le 26 mai 1978, pour approuver ce que l'on a appelé à l'époque, « les principes de la protection du front intérieur et de la paix sociale ». Ces principes, que le peuple égyptien a approuvés par une majorité de 98,29\%, ont été votés ultérieurement par l'Assemblée du peuple sous forme de loi $n^{\circ} 33$ de 1978. Par ce référendum, le président Sadate fit accepter par le peuple égyptien la réglementation de la vie politique et la dégradation des droits politiques de « tous ceux qui [avaient] perverti la vie politique d'avant la Révolution de $1952 »^{53}$.

43 L'article 152 prévoit que le président de la République «peut » consulter le peuple. Il s'agit donc d'un référendum facultatif pour celui-ci ${ }^{54}$. La comparaison de la solution apportée par cet article avec celle retenue par les constitutions précédentes montre à quel point le président de la République est libre de déterminer l'objet et le moment de la consultation. Aucun rôle n'est reconnu ni au Parlement, titulaire de la compétence législative générale, ni au gouvernement que la Constitution associe pourtant au président de la République pour l'élaboration de la politique générale de l'État. Les articles 145 de la Constitution de 1956 et 129 de celle de 1964 donnaient, cependant, au président de la République la faculté de déclencher ce type de consultation après " avis conforme de l'Assemblée nationale ${ }^{55}$. Aujourd'hui, le président de la République est seul à déterminer l'opportunité de la décision de mise en jeu de la procédure de l'article 152 et, également, le moment de cette décision. Ainsi, s'il s'agit d'un projet de loi ou d'un traité, que le président estime important, ce dernier peut, avant que le Parlement en soit saisi ou après le vote du projet, soumettre le texte au référendum afin de 
connaître le sentiment populaire ${ }^{56}$. La pratique égyptienne confirme cette solution. La loi $\mathrm{n}^{\circ} 33$ de 1978, sur la protection du front intérieur, avant d'être votée par le Parlement, fut approuvée par référendum le 21 mai de la même année. Quant au traité égypto-israélien, il a été d'abord approuvé par l'Assemblée du peuple puis soumis au référendum ultérieurement.

\section{La mainmise du chef de l'État sur la procédure législative}

Parlement, quant à lui, n'est qu'une chambre d'enregistrement. Cette situation est due à plusieurs raisons - nous en avons exposé quelques unes - et qui font de la procédure législative parlementaire une simple et belle promenade pour le gouvernement. En effet, dans la procédure d'élaboration de la loi, les Constitutions de l'Égypte républicaine ainsi que les règlements intérieurs des assemblées, ont mis à la disposition du pouvoir exécutif quelques prérogatives de nature à réduire considérablement la portée de l'intervention du Parlement.

D'après l'article 109 de la Constitution égyptienne actuelle, l'initiative des lois appartient au "président de la République et à chaque membre de l'Assemblée du peuple[...] ». L'article 28 de la Constitution monarchique de 1923 disposait à cet égard que « l'initiative des lois appartient au roi, au Sénat et à la Chambre des députés». Sous la Constitution de l'époque monarchique ${ }^{57}$, comme sous celles de l'époque républicaine ${ }^{58}$, une distinction est nettement faite entre la procédure d'examen des projets émanant du gouvernement et celle applicable aux propositions provenant d'une initiative parlementaire. En effet, les projets sont directement soumis au président de l'Assemblée avant d'être renvoyés à la commission spécialisée ou d'être soumis à l'Assemblée ${ }^{59}$, tandis que les propositions, avant d'arriver à cette commission, sont strictement examinées. Elles sont soumises à ce que nous pouvons qualifier de "contrôle politique a priori de constitutionnalité », exercé par les "commissions d'initiative " des assemblées de 1923 à 1952, et actuellement par la commission «d'initiatives et des plaintes au sein de l'Assemblée du peuple ${ }^{60}$ ».

Actuellement, à l'intérieur de l'Assemblée du peuple, on peut distinguer trois types de commissions parlementaires: les commissions permanentes, les commissions de contrôle et les commissions d'enquête. Les commissions permanentes sont régies par les articles 36 à 81 du règlement intérieur de l'Assemblée du peuple. Il s'agit, d'après l'article $36 \mathrm{du}$ règlement de l'Assemblée, de 18 commissions. Une commission permanente est composée, d'après l'article 37 du règlement intérieur de l'Assemblée, d'un nombre de membres déterminé, au début de chaque législature ordinaire, par l'Assemblée sur l'initiative de son bureau. Le bureau, quant à lui, est composé, d'après l'article 10 du règlement intérieur, du président de l'Assemblée et de ses deux viceprésidents, élus au début de chaque session ordinaire annuelle à la majorité relative des membres de l'Assemblée.

D'une manière générale, d'après le règlement intérieur de l'Assemblée du peuple, le travail d'une commission permanente spécialisée consiste à examiner le projet de loi et à présenter un rapport à son sujet à l'Assemblée. La commission présente les articles du projet de la loi sous forme de tableau comparatif comprenant d'une part, le texte des articles tel qu'il a été rédigé par le gouvernement, et d'autre part le texte proposé par la

Égypte/Monde arabe, 2 | 2005 
commission contenant les amendements. Conformément à l'article 146/3 du règlement intérieur de l'Assemblée du peuple, le débat parlementaire s'effectue sur la base du texte présenté par la commission. Les commissions parlementaires ne relèvent jamais les défauts des projets présentés par le gouvernement. Pour cette raison, les débats parlementaires se déroulent sur des projets qui n'expriment en principe que le point de vue $\mathrm{du}$ gouvernement. Cela explique le nombre considérable d'exceptions d'inconstitutionnalité soulevées contre les lois en vigueur. En effet, qualifiées de «tremplins qui permettent à des personnalités politiques d'accéder à de hautes fonctions gouvernementales ${ }^{61}$ », les commissions permanentes sont soumises aux pressions de l'exécutif qui s'exercent dans le sens de l'adoption de rapports favorables aux projets d'origine gouvernementale. À aucun moment, dans ces rapports, les membres des commissions ne font apparaître les carences des projets de lois qui donnent souvent lieu à des modifications ultérieures. C'est le cas, par exemple, du projet de loi sur les investissements étrangers et les zones franches en $1973^{62}$. Cela explique également le nombre très élevé de décisions d'inconstitutionnalité prononcées ultérieurement par la Haute Cour constitutionnelle. Entre 1970 et fin juin 1991, la Cour constitutionnelle a ainsi enregistré 192 exceptions d'inconstitutionnalité soulevées par les différentes juridictions à côté de quelque 183 recours en interprétation et autres ${ }^{63}$.

D'une manière générale, la Constitution égyptienne de 1971 semble vouloir soumettre la procédure de discussion et de vote de la loi à la stricte surveillance du gouvernement. En effet, qu'il s'agisse de projets de lois ordinaires, de projets de lois de finances ou de projets de lois d'autorisation de ratification des traités internationaux, les ministres ont pour mission de défendre, chacun dans son domaine, le point de vue de l'exécutif afin d'obtenir l'adoption de ces projets, et, à cet effet, ils doivent, d'après l'article 135 de la Constitution, "être entendus par l'Assemblée du peuple et ses commissions toutes les fois qu'ils demandent la parole ». Ainsi examinés et préparés en commission, conformément aux souhaits du gouvernement, le projet de loi et le rapport qui lui est joint sont transmis à l'Assemblée pour être adoptés à la majorité simple des membres présents. Dans cette phase également, les projets provenant de l'exécutif bénéficient, pour être adoptés, de la pression gouvernementale sur les députés.

Les pouvoirs des parlementaires, dans la procédure actuelle du vote de la loi, sont, eux, considérablement limités, alors que, sous le régime de la Constitution monarchique de 1923, le droit d'amendement fut largement reconnu aux parlementaires par les règlements intérieurs des assemblées. Un amendement pouvait émaner d'un ou de plusieurs membres du Parlement. Les parlementaires pouvaient proposer de modifier profondément un projet non encore voté et rien n'empêchait même de proposer un amendement qui soit un véritable contre-projet ${ }^{64}$. Le droit d'amendement est actuellement reconnu par l'article 140 du règlement de l'Assemblée du peuple et se trouve expressément limité, tant en ce qui concerne les projets de lois de finances que pour certains projets de décrets-lois déposés par le président de la République, ainsi que pour les projets de lois d'autorisation de ratification des traités internationaux.

50 L'intervention de l'Assemblée du peuple pour l'approbation du plan général de développement économique et sociale, du budget général de l'État et des comptes définitifs du budget de l'État est prévue par les articles de 114 à 118 de la Constitution de 1971. La procédure législative applicable à ces matières est qualifiée par le règlement intérieur de l'Assemblée ${ }^{65}$ de "procédure d'urgence». Elle constitue donc une 
exception à la procédure législative ordinaire. Le projet de budget général de l'État est évidemment d'origine gouvernementale. Il est soumis - d'après l'article 115 de la Constitution - à l'Assemblée du peuple deux mois au moins avant le commencement de l'Exercice financier. Son origine gouvernementale lui fait bénéficier de la distinction déjà étudiée entre les propositions provenant des parlementaires et les projets gouvernementaux ${ }^{66}$, notamment de la procédure exceptionnelle prévue par le règlement intérieur de l'Assemblée. En effet, dès qu'il est reçu par l'Assemblée du peuple, le projet du budget est transmis d'urgence à «la commission du plan et du budget» par le président de l'Assemblée. Cette dernière en est informée par son président à la première réunion qu'elle tiendra ${ }^{67}$. Ce projet est discuté d'urgence par l'Assemblée et bénéficie de toutes procédures d'urgence prévues par son règlement intérieur ${ }^{68}$. D'après le second alinéa de l'article 115 de la Constitution, l'Assemblée du peuple ne peut apporter de modifications au budget général de l'État qu'avec le consentement $\mathrm{du}$ gouvernement. Cette restriction du droit d'amendement est également prévue par l'article 130 du règlement intérieur de l'Assemblée du peuple. Cette solution entraîne le mécontentement de certains publicistes égyptiens qui y voient une limitation injustifiée du pouvoir financier de l'Assemblée du peuple ${ }^{69}$.

51 Nous avons déjà vu que le président de la République intervient dans le domaine de la compétence législative de l'Assemblée du peuple par le moyen de décrets-lois conformément aux article 74, 108 et 147 de la Constitution de 1971. Une fois édictés, ces décrets-lois sont soumis à l'Assemblée du peuple de la manière déjà étudiée ${ }^{70}$. Ils ne représentent pas seulement une exception à la compétence législative générale de l'Assemblée du peuple, puisque la procédure utilisée par l'Assemblée en vue de leur approbation constitue, elle aussi, une exception à la procédure législative ordinaire. Cette procédure exceptionnelle est prévue par les articles 173 à 177 de son règlement intérieur. En effet, conformément à l'article 173, ces décrets-lois sont directement transmis - par le président de l'Assemblée - aux commissions permanentes pour avis ${ }^{71}$. Leur examen par les commissions est prioritaire par rapport à tout autre sujet. L'Assemblée peut également - à l'initiative de son président - soumettre les décrets d'importance particulière à la commission générale de l'Assemblée ${ }^{72}$ ou à une commission spéciale formée à cette fin et dont les membres sont librement choisis par le président de l'Assemblée ${ }^{73}$.

52 Le caractère exceptionnel de la procédure législative d'approbation des décrets-lois se manifeste à plusieurs niveaux. D'une part, d'après l'article $174 \mathrm{du}$ règlement intérieur de l'Assemblée du peuple, les membres de celle-ci ont l'interdiction formelle de formuler des propositions d'amendement des dispositions des décrets-lois édictés par le président de la République sur la base des articles 74 et 108 de la Constitution. Les parlementaires n'ont, en effet, que la possibilité d'admettre ces décrets ou de les rejeter tels qu'ils sont. En cas de rejet, le président de la République est informé des détails de la procédure suivie. D'autre part, l'article 176 du règlement intérieur de l'Assemblée du peuple ne semble pas interdire aux parlementaires de formuler des propositions d'amendement des dispositions des décrets-lois édictés sur la base de l'article 147 de la Constitution. Cependant, l'article 176 traite ces propositions d'amendement de la même manière que les propositions de lois présentées par les parlementaires. Par conséquent, toute proposition d'amendement est transmise à la «Commission d'initiatives et des plaintes ", qui juge si elles méritent ou non d'être prises en considération par la commission permanente spécialisée, et ensuite par l'Assemblée ${ }^{74}$. Il s'agit, en fait, d'une procédure longue et compliquée qui n'apparaît pas compatible avec la procédure 
législative accélérée relative aux décrets "de nécessité " de l'article 147 de la Constitution. En tous cas, si l'Assemblée décide de rejeter les décrets-lois de l'article 147 de la Constitution (une chose qui n'a jamais eu lieu), elle doit - conformément aux articles 147 de la Constitution et 175 du règlement de l'Assemblée - «en approuver l'exécution pour la période précédente ou d'en régler autrement les effets ».

Venons-en, maintenant, à la conclusion d'un traité international qui comprend, on le sait, trois phases différentes : la négociation, la signature et la ratification. Le chef de l'État en Égypte a traditionnellement le droit de conclure les traités, c'est donc à lui qu'a toujours appartenu la compétence pour négocier, signer et ratifier. Cependant, pour la ratification de certaines catégories de traités, le principe de l'intervention unique du chef de l'État est assorti de quelques exceptions ou plutôt de quelques tempéraments. Ainsi, l'article 151 de la Constitution égyptienne de 1971 prévoit que :

Le président de la République conclut les traités et les communique à l'Assemblée du peuple accompagnés d'un exposé adéquat. Les traités ont force de loi après leur conclusion, leur ratification et leur publication selon les règles établies. Toutefois les traités de paix, d'alliance, de commerce et maritime, ainsi que tous les traités qui entrânent une modification du territoire de l'État, qui se rattachent aux droits de souveraineté ou qui grèvent le Trésor de l'État de dépenses non prévues au budget, doivent recevoir l'approbation de l'Assemblée du peuple.

Comme pour les lois de finances, la loi d'autorisation de ratification des traités est d'origine gouvernementale. Elle est entièrement préparée par le pouvoir exécutif. Pour la ratification d'un traité, le droit d'amendement se voit considérablement limité, voire interdit. En effet, il ne s'agit plus, pour les parlementaires, de pouvoir demander l'accord du gouvernement pour apporter des modifications au projet du traité. Ce dernier ne peut être modifié qu'après de nouvelles négociations entre les autorités égyptiennes et la puissance étrangère contractante. Cette solution, traditionnelle en droit égyptien, comme d'ailleurs dans la plupart des systèmes juridiques contemporains $^{75}$, fut d'abord consacrée par les règlements intérieurs des chambres sous la Constitution de l'Égypte monarchique ${ }^{76}$. L'article 87 du règlement intérieur de la Chambre des députés prévoyait, jusqu'en 1952, que :

Lorsque l'Assemblée est saisie d'un projet de loi par lequel le gouvernement lui demande l'approbation d'un traité conclu avec une puissance étrangère, il ne lui est pas permis d'y apporter des amendements. Elle décide de l'approbation, du rejet ou de l'ajournement. En ce dernier cas, l'Assemblée appelle l'attention du gouvernement sur les clauses du traité qui ont motivé sa décision.

Ainsi ne pouvait-on procéder au vote sur les articles du traité ni en amender le texte. De plus, le Parlement, lors de l'examen d'un traité, devait tenir compte des délais impartis pour la ratification ${ }^{77}$. La même solution est actuellement consacrée par l'article 197/2 du règlement intérieur de l'Assemblée du peuple.

\section{L'insuffisance du travail parlementaire}

Deux traits essentiels caractérisent la procédure de discussion et de vote des lois dans les parlements de l'Égypte républicaine. D’abord, la brièveté des débats et, ensuite, la majorité écrasante dont bénéficient les lois d'origine gouvernementale ou en provenance des parlementaires de la majorité. L'insuffisance des débats et la rapidité de vote sont, à notre avis, due à deux raisons principales. D'abord, la carence de l'effort 
personnel des parlementaires, puis le monocaméralisme de fait, dont l'un des inconvénients est la légèreté de la procédure législative.

Nous avons déjà exposé les divers facteurs qui sont de nature à diminuer la portée du contrôle du Parlement égyptien sur l'action de l'exécutif. Ces facteurs tiennent essentiellement à la domination du Parlement par le chef de l'État, irresponsable politiquement et principal acteur de la politique générale de l'État ${ }^{78}$. Pendant la phase du vote des lois, le Parlement se voit accorder une dernière chance pour affirmer son rôle de titulaire de la compétence législative générale. Cette chance n'est pourtant jamais saisie. Cela est essentiellement dû à la composition du Parlement et à la volonté des parlementaires eux-mêmes. En effet, selon la « règle démocratique » consacrée par les constitutions de l'Égypte républicaine, la moitié des membres de l'Assemblée du peuple doit être choisie parmi les ouvriers et les paysans ${ }^{79}$. Ainsi, la moitié des parlementaires ne possède pas les compétences minimales requises pour discuter des délicats problèmes techniques liés au processus législatif. Leur rôle se limite à légitimer a posteriori l'action législative de l'exécutif. La seconde moitié des parlementaires pourrait être facilement classée en deux catégories. D'une part, les membres du parti au pouvoir, majoritaires, totalement soumis au chef de l'État et dont le rôle est d'appuyer, sans réserve, l'action du gouvernement ou de lui adresser des critiques non déclarées ${ }^{80}$ et, d'autre part, les parlementaires de l'opposition, minoritaires et seuls intéressés à critiquer publiquement la politique du gouvernement. Leur critique est cependant sans suite, car leur poids parlementaire n'est pas de nature à influencer les orientations du gouvernement.

Comme nous l'avons vu, le Parlement actuel de l'Égypte est composé depuis 1971 de l'Assemblée du peuple à laquelle a été ajoutée, en 1980, une seconde chambre à compétence purement consultative et qui ne participe ni à la fonction législative ni à celle de contrôle. Nous constatons donc que le Parlement actuel est monocaméral; il se compose effectivement de la seule "Assemblée du peuple». En Égypte, c'est la Constitution de 1956 qui introduisit le système monocaméral, mettant ainsi fin à vingtneuf ans de bicaméralisme réel (de 1923 à 1952). Les partisans du monocaméralisme s'appuyaient essentiellement sur la doctrine française de la souveraineté nationale. Pour eux, l'Égypte est devenue une République, par conséquent il n'y existe plus d'aristocrates et il ne doit y avoir qu'une seule représentation, celle de la nation souveraine. La loi ne peut donc être que l'expression de la volonté générale exprimée par la majorité des électeurs. Ils estimaient, en outre, que la période révolutionnaire exigeait un système monocaméral pour exécuter les réformes et empêcher toute lenteur dans la préparation des lois. D'après eux, ce système devait enfin permettre d'éviter la réapparition en Égypte d'une aristocratie nouvelle ${ }^{81}$.

Il est vrai que les circonstances de l'après-révolution exigeaient la réalisation de réformes approfondies et rapides dans tous les domaines de la vie des Égyptiens. Mais il faut reconnaître également que, pour éviter la lenteur de la procédure législative, on en est arrivé à réduire le rôle du Parlement à n'être qu'un simple «enregistreur » de la volonté de l'exécutif. Ce trait demeure l'une des caractéristiques du Parlement égyptien de 1952 à nos jours. Un simple regard sur le nombre de législations existantes actuellement en Égypte et les modifications qui y ont été apportées ainsi que sur les décisions d'inconstitutionnalité montre à quel point le monocaméralisme, à côté de la pression gouvernementale, entraîne l'accélération de la procédure législative et par conséquent l'existence d'un phénomène redoutable de "diarrhée législative ${ }^{82}$. On 
estime, en effet, qu'il existait en Égypte jusqu'en 1991, 7681 lois et 40057 décrets présidentiels et arrêtés ministériels ${ }^{83}$. Pour ne prendre que deux exemples des nombreuses modifications que peut subir une seule loi, nous citerons la loi $\mathrm{n}^{\circ} 210$ de 1951 sur les fonctionnaires de l'État, modifiée à 88 reprises jusqu'à son abrogation en $1954^{84}$, et la loi $\mathrm{n}^{\circ} 38$ de 1972 sur l'Assemblée du peuple qui a subi 11 modifications jusqu'en $1990^{85}$. Et, comme nous l'avons déjà signalé, entre 1970 et 1991, la Cour constitutionnelle avait enregistré 192 exceptions d'inconstitutionnalité soulevées par les différentes juridictions à côté de quelques 183 recours en interprétation et autres. De même que pour les lois internes, les projets de traités internationaux ne sont pas suffisamment discutés par l'Assemblée et les autorisations parlementaires accordées au chef de l'État afin de les ratifier sont très facilement votées. Il suffit, par exemple, de signaler que dans sa $58^{\text {e }}$ séance, en date du 21 mai 1990, l'Assemblée du peuple a autorisé, en 45 minutes, la ratification de 29 traités, consacrant ainsi une minute et demi de discussion et de vote à chaque traités ${ }^{86}$.

\section{BIBLIOGRAPHIE}

ABÛ-ZAYD ALî M., 1993, Le Bicaméralisme et son rôle dans la réalisation de la démocratie, le Caire.

Ameler Michel, 1986, Les Parlements dans le monde, Bruxelles, Bruylant.

'AMER A., 1955, Les Causes de la faillite du système constitutionnel égyptien (1923-1952), Thèse, Paris.

'AsFûR S., 1980, Le Régime constitutionnel égyptien, Alexandrie.

EL-SAYYID SABRî, 1969, Les Règlements législatifs, Le Caire, 1969.

Fанмî 0. H., 1980, La Fonction législative du chef de l'État dans les régimes présidentiel et parlementaire, Le Caire.

Hassabo A., 1978, La Théorie des circonstances exceptionnelles, thèse, Le Caire.

- 1989, Les Rapports entre le pouvoir législatif et le pouvoir exécutif en France et en Égypte, Thèse, Clermond-Ferrand.

IвRÂHÎM SHUKRî Muhammad, 1925, Les Règlements intérieurs des chambres en Égypte, thèse, Paris. cIzz al-DiN G.,1963, Le Système unicaméral, son application en Égypte et en Syrie, Genève.

JACQUEMOND R., 1988, « La Haute Cour constitutionnelle égyptienne et le contrôle de la constitutionnalité des lois », AIJC, IV.

KAMÂL F., 1972, Les Dispositions parlementaires, Le Caire.

Luchaire F., 1987, « article 38 », La Constitution de la République française, Paris, Economica.

MIREL P., 1981, Recherches sur le système politique égyptien, Thèse, Poitiers.

Moнsin Khalîl, s. d., Le Régime constitutionnel égyptien, Alexandrie. 
NASIF 'A., 1981, La Portée de l'équilibre entre le pouvoir politique et la responsabilité dans l'État moderne, Thèse, Le Caire.

Plouvin J.Y., 1980, Le Droit présidentiel de demander une nouvelle délibération de la loi, R.D.P.

SÂLıH I. A., 1966, Le Régime politique de la République arabe unie et la notion de démocratie, Thèse, Paris.

SALLÂM I. Z., 1982, Le Contrôle politique sur les actes du pouvoir exécutif dans le régime parlementaire, Thèse, le Caire.

SHÛKRI N., 1929, La Constitution égyptienne et le contrôle de constitutionnalité des lois, Thèse, Paris.

Томісне J., 1960, « L'Union nationale, parti unique de la République arabe Unie », La Documentation française, $\mathrm{n}^{\circ} 2632$.

\section{NOTES}

1. Sur les différents moyens du contrôle parlementaire en Égypte, voir : 'AAmr Hassabo, 1989, p. 376 et sq. ; Ihâb Zakî Sallâm, 1982, p. 23 et sq. ; Pierre Mirel, 1981, p. 325 et sq.

2. Pour plus de détails sur les différentes formations politiques d'avant 1952, ainsi que pour le fonctionnement du système partisan de l'Égypte monarchique, voir en particulier Adel cAmer, 1955.

3. Sur l'organisation et le fonctionnement du parti unique en Égypte de 1952 à 1976, voir : Tomiche, 1960 ; Ibrâhîm 'Aly Sâlih, 1966, p. 451 et sq. ; Mirel, id., p. 144 et sq. ; Hassabo, id., p. 324 et $s q$.

4. Voir sur ce point : Mirel, id., p. 165 et sq. ; Hassabo, id., p. 358 et sq.

5. Hassabo, id., p. 328, 345 et 444.

6. Voir les articles 38 de la Constitution de 1923, 111 de la Constitution de 1956 et 91 de celle de 1964.

7. Rappelons que la disposition concernant l'accord de la population par référendum ne figurait dans aucune des constitutions égyptiennes précédentes. La consultation populaire prévue par l'article 136 s'inscrit dans le cadre de la place sans précédent qu'accorde la Constitution de 1971 au référendum.

8. Voir infra.

9. Hassabo, id., p. 184-185.

10. Mirel, id., p. 261.

11. Id. p. 161 et $s q$.

12. Voir infra.

13. Articles 67 de la Constitution de 1956 et 49 de celle de 1964 .

14. Sucâd ${ }^{\mathrm{c} A s f u ̂ r, ~ 1980, ~ p . ~} 170$.

15. Hassabo, id., p. 150.

16. Hassabo, id., p. 153-154.

17. Nagîb 1929, p. 139 et sq.

18. Hassabo, id., p. 247.

19. Plouvin, 1980 , p. 1573.

20. Voir également l'article 130 de la Constitution de 1965 et 22 de celle de 1964.

21. 'Asfûr, id., p. 87.

22. Voir les articles 38/1 et 84 de la Constitution de 1964.

23. Voir les articles 90/2 et 84 de la Constitution de 1964.

24. Voir dans ce sens : Nâsif, 1981, p. 388 ; Pierre Mirel, id., p. 328, note 1; Hassabo, id., p. 491. 
25. Il est à noter, à cet égard, que la question de confiance était consacrée par l'article 88 de la Constitution de 1964 qui prévoyait que «Le Premier ministre peut demander un vote de confiance de l'Assemblée en faveur de son gouvernement lors de l'exposé de son programme ou à l'occasion de tout autre exposé du gouvernement concernant la politique générale de l'État ».

26. Mirel, id., p. 328.

27. Il faut noter à cette occasion que ces obstacles pour la mise en jeu de la responsabilité politique du gouvernement ne figuraient pas dans l'article 90 de la Constitution de 1964, qui prévoyait à cet effet qu'à la suite d'» une motion de censure par l'Assemblée, le Premier ministre doit présenter la démission de son gouvernement au président de la République [...]».

28. Mirel, id., p. 329.

29. Fahmî, 1980, p. 380 et sq. ${ }^{c}$ Asfûr, id., p. 163.

30. Voir également les articles 136 de la Constitution de 1956 et 120 de celle de 1964.

31. 'Asfûr, id., p. 137.

32. Gamal al-Dîn, 1982, p. 242.

33. Fahmî, id., p. 387. ; Hassabo, id., p. 97.

34. 'Asfûr, id., p. 139-140.

35. Fahmî, id., p. 391-392.

36. Gamâl al-Dîn, id., p. 163 ; Fahmî, id., p. 384-385.

37. L'article 108, diffère, à cet égard, de l'article 38 de la Constitution française de 1958, dont la mise en œuvre n'exige pas l'existence de circonstances de ce genre. Le gouvernement français peut demander l'application de cet article au cas où il le jugerait nécessaire pour l'exercice de son programme et sous certaines conditions. Voir à ce propos Luchaire, 1987, p. 795 et sq.

38. Hassabo, id.,p. 95.

39. Cette expression est reconduite d'une constitution égyptienne à l'autre depuis celle de 1923.

40. Voir en particulier : Gamâl al-Dîn, id., p. 65 et sq. Hassabo, 1978, p. 104-105; Ahmad Medhat ${ }^{c}$ Alî, id., p. 106 et sq.

41. Hassabo, id., p. 105.

42. Pour ces deux types de contrôle de légalité et de constitutionnalité voir : Gamâl al-Dîn, id., p.

71 et sq. ; Hassabo, id., p. 109-110.

43. El-Sayyid Sabrî, 1969, p. 37-38.

44. Gamâl al-Dîn, id., p. 78 et sq.

45. Id., p. 84 et $s q$.

46. Hassabo, id., p. 106.

47. Mirel, id., p. 260.

48. Pour une étude détaillée de l'article 74, voir: Gamâl al-Dîn, id., p. 113 et sq.; Hassabo, id., p. 110 ; Mâhir Nadar, id., p. 321 et sq. ; Mirel, id., p. 266 et sq.

49. Voir également les articles 145 de la Constitution de 1956 et 129 de celle de 1964.

50. Mâhir Nadar, id., p. 430.

51. Id., p. 433-434.

52. Hassabo, id., p. 91 et pour plus de détails voir Mâhir Nadar, id., p. 439 et sq.

53. Pour plus de détails voir Mâhir Nadar, id., p. 443 et sq.

54. Id., p. 430-431.

55. Id., p. 229.

56. Id., p. 436.

57. Ibrâhîm Shukrî, 1925, p. 109 et ss.

58. 'Asfûr, id., p. 240 et sq. ; Hassabo, id., p. 207 et sq.

59. Article $140 \mathrm{du}$ règlement intérieur de l'Assemblée du peuple.

60. Article 163 du règlement intérieur de l'Assemblée du peuple.

61. Mirel, id., p. 317.

62. Id. p. 317. 


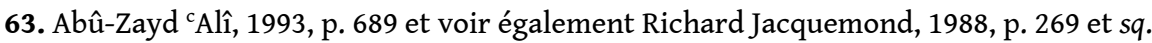

64. Ibrâhîm Shukrî, id., p. 112

65. Section I du chapitre 2 du titre 6 du règlement intérieur de l'Assemblée du peuple.

66. voir supra.

67. Article $126 \mathrm{du}$ règlement intérieur de l'Assemblée.

68. Article $127 \mathrm{du}$ règlement intérieur de l'Assemblée

69. Mohsin khalîl, s. d., p. 365-366 ; Hassabo, id., p. 243.

70. Voir supra.

71. Les Commissions parlementaires permanentes sont au nombre de 18 , voir supra.

72. La Commission générale de l'Assemblée est composée - conformément l'article $21 \mathrm{du}$ règlement intérieur de l'Assemblée - ainsi : 1- le président de l'Assemblée (président) ; 2- les deux vice-présidents de l'Assemblée; 3- les présidents des commissions permanentes; 4- les représentants des groupes parlementaires à l'Assemblée ; 5 - cinq membres de l'Assemblée choisis par le président de l'Assemblée, parmi lesquels un indépendant, si le nombre des membres indépendants est supérieur à dix au moins.

73. La commission spéciale est - d'après l'article 82 du règlement intérieur de l'Assemblée autorisée par l'Assemblée sur l'initiative de son président ou du gouvernement. Les membres de cette commission sont librement choisis par le président de l'Assemblée.

74. Voir supra à propos de la distinction des projets de loi d'origine gouvernementale et les propositions de lois présentées par les parlementaires au niveau de la procédure législative.

75. Ameler, 1986. p. 1446-1447.

76. Ibrâhîm Shukrî, id., p. 72-73 ; Fu'âd Kamâl, 1972, p. 146.

77. Ibrâhîm Shukrî, id., p. 72-73.

78. Voir supra.

79. Article 78 de la Constitution de 1971 et I de la loi nº 38 de 1972 sur l'Assemblée du peuple.

80. Sallam, id., p. 210 ; Abû-Zayd Alî, id., p. 683.

81. Sur ce point : 'Izz al-Dîn, 1963 p. 59 et sq.

82. Voir Abû-Zayd Alî, id., p. 684 et sq. et les nombreux auteurs cités par lui.

83. Abû-Zayd Alî, id., p. 684 , note 2 .

84. Id., p. 685-686, note 3 .

85. Id., p. 687-688, note 1.

86. Le Rapport stratégique arabe, Centre de recherches politiques et stratégiques, 1991, p. 390-391.

INDEX

Mots-clés : Constitution, pouvoir exécutif, pouvoir législatif

\section{AUTEUR}

EID AHMED EL-GHAFLOUL

Faculté de droit de Benha, Université de Zagazig (Égypte) 